\title{
THE EVOLUTION OF TWO STACKS IN BOUNDED SPACE AND RANDOM WALKS IN A TRIANGLE
}

\author{
Philippe FLAJOLET \\ INRIA \\ Rocquencourt \\ 78150 Le Chesnay (France)
}

\begin{abstract}
We analyse a simple storage allocation scheme in which two stacks grow and shrink inside a shared memory area. To that purpose, we provide analytic expressions for the number of 2-dimensional random walks in a triangle with two reflecting barriers and one absorbing barrier.

We obtain probability distributions and expectations of characteristic parameters of that shared memory scheme, namely the sizes of the stacks and the time until the system runs out of memory.

This provides a complete solution to an open problem posed by Knuth in "The Art of Computer Programming", Vol. 1, 1968 [Ex. 2.2.2.13].
\end{abstract}

\section{Introduction}

The analysis of dynamic data structures is often the source of intriguing mathematical questions. As opposed to classical average case of algorithms like sorting, searching etc .... the difficulty lies in understanding algorithmic behaviours that are inherently dynamic. This sometimes requires introducing new probabilistic or combinatorial models, that are of independent interest.

This paper studies what is perhaps the simplest storage allocation algorithm. Assume that two stacks are to be maintained inside a shared (contiguous) memory area of a fixed size $m$. A trivial algorithm will let them grow from both ends of that memory area until their cumulated sizes fill the initially allocated storage ( $m$ cells), and the algorithm stops having exhausted its available memory.

That shared storage allocation algorithm is to be compared to another option, namely allocating separate zones of size $m / 2$ to each of the two stacks. This separate storage allocation method will then halt as soon as any one of the two stacks reaches size $m / 2$.

Several measures may be introduced to compare these two schemes. One of them is the expected number of operations that can be treated by the algorithms under some appropriate probabilistic model. Another interesting measure of the efficiency of the shared allocation algorithm that was proposed by Knuth [7], is the expected size of the largest stack when both stacks meet and the algorithm runs out of storage: if that quantity is close to $m / 2$, then little benefit should result from sharing storage while if it differs appreciably from $m / 2$ then the process of sharing memory proves advantageous. 
It is our purpose here to completely analyse this problem and thus solve a question posed by Knuth in 1968 in [7]. Partial results have been obtained earlier by Yao [11], but it appears that covering all cases of the original problem cannot be achieved by an extension of Yao's methods.

Before going to more technical details, one may wish to contrast our treatment with other dynamic analyses of algorithms.

a. The analysis of simple stacks under sequences of operations has been undertaken by Knuth in [7]. Corresponding techniques are essentially enumerative and belong to what Riordan calls the "Catalan domain" where the Catalan numbers

$$
C_{n}=\frac{1}{n+1}\left(\begin{array}{c}
2 n \\
n
\end{array}\right)
$$

play a crucial role. The evolution of a single stack inside a fixed memory area is studied under a different form (viz. the distribution of height in planar trees) by [1].

b. Binary search trees were later analysed in a particular dynamic context by Jonassen and Knuth [6]. The title of their paper ("A Trivial Algorithm whose Analysis is Not") reveals some of the intricacies of the analysis where Bessel functions appear.

c. Francon in [5] showed interesting results concerning the analysis of binary search trees (as well as other comparison based structures) when considering the set of all possible sequences of operations up to order isomorphism. The results were later extended in [4] using continued fraction techniques of Flajolet [3]. Part of our treatment relies on techniques of [3] initially motivated by the work of [1].

d. Returning to storage allocation algorithms, the buddy system was partially analysed by Purdom. As already mentioned the shared storage allocation algorithm was studied by Yao [11] who obtained results that correspond to what we call here the expanding case. Our results cover all cases and also lead us to more general distribution estimates of the various parameters involved.

As has been noticed since the problem was initially posed by Knuth, the natural formulation is in terms of random walks [9]. Here the random walk takes place in a triangle in a 2 -dimensional lattice space: a state is the couple formed with the size of both stacks; the random walk has two reflecting barriers along the axes (a deletion takes no effect on an empty stack) and one absorbing barrier parallel to the second diagonal (the algorithm stops when the combined sizes of the stacks exhaust the available storage).

It may be of interest to note that random walk problems bearing some resemblance to ours appear in queuing theory when studying coupled queues. In particular Flatto, Fayolle and lasnogorodski use in this context conformal mapping techniques to solve a functional equation problem essentially equivalent to a 2-dimensional walk with two reflecting barriers. (See also Cohen's book on random walks and boundary value problems for some related issues.) It does not seem that their techniques apply here because of the presence of a third absorbing barrier. However, it may be of interest to notice that while their solutions involve elliptic integrals, the expressions we obtain in one of the cases (the "metastable case") lead to functions of the form

$$
\varphi(x ; q)=\sum_{n \geq 1} \frac{x^{n}}{1-q^{n}}
$$



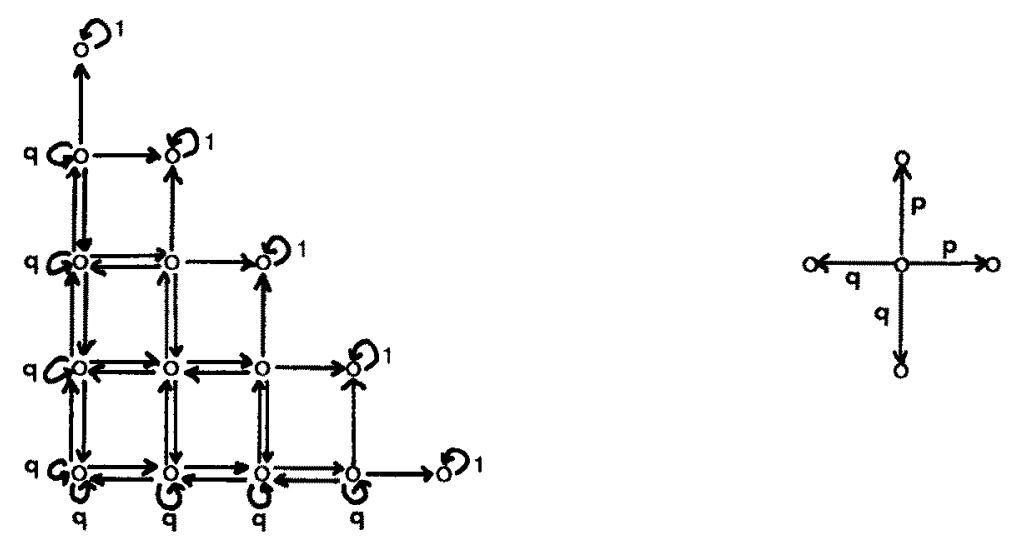

Figure 1: The Markov chain modelling the shared storage allocation algorithm (when $m=4$ ).

also ciearly related to elliptic functions.

Two-dimensional counting problems otherwise occur in statistical mechanics, most notably in the study of the Ising model (see e.g. [8]). It is another wonder that the common denominator for expressions of probabilities obtained here in the metastable case coincides with expressions occurring in the solution of the dimer problem of statistical mechanics.

We now proceed with precise definitions and a more detailed presentation of the contents of the paper.

We consider two stacks coexisting in memory by growing and shrinking inside a shared memory area of size $m$ with memory cells numbered by the integers [1..m]. A 2-stack history is an infinite word over the alphabet:

$$
\left\{I_{1}, I_{2}, D_{1}, D_{2}\right\}
$$

where $I_{1}$ represents an insertion on stack $1, D_{1}$ a deletion from stack 1 (this has no effect if stack 1 is empty), and $I_{2}, D_{2}$ are similar operations performed on stack 2.

At any stage, stack 1 occupies memory locations [1.x] for some integer $x$ (if $x=0$, then stack 1 is empty), and stack 2 occupies locations $[m-y+1 . . m]$ for some $y$ ( $y$ is thus the size of stack 2 , and if $y=0$ stack 2 is empty), with the obvious constraint $x+y \leq m$. Initially both stacks are empty so that $x=y=0$. The state of the system at any time is described by the couple of integers $(x, y)$; the effect of operations $I_{1}, I_{2}, D_{1}, D_{2}$ is to move from a state $(x, y)$ to a new state $\left(x^{\prime}, y^{\prime}\right)$, where $\left(x^{\prime}, y^{\prime}\right)$ is determined by the rules:

$I_{1:}\left(x^{\prime}, y^{\prime}\right)=(x+1, y)$ if $x+y<m,(x, y)$ otherwise;

$D_{1:}\left(x^{\prime}, y^{\prime}\right)=(x-1, y)$ if $x>0, x+y<m,(x, y)$ if $x+y=m,(0, y)$ otherwise;

$I_{2:}\left(x^{\prime}, y^{\prime}\right)=(x, y+1)$ if $x+y<m,(x, y)$ otherwise;

$D_{2:}\left(x^{\prime}, y^{\prime}\right)=(x, y-1)$ if $y>0, x+y<m,(x, y)$ if $x+y=m,(0, y)$ otherwise.

Thus operations have no effect after a state $(x, y): x+y=m$ has been reached, when the algorithm runs out of memory and stops. In this case, we 
say that overflow or absorption has occurred.

One can see that the set of histories such that no overflow occurs has measure 0 . The problem asked by Knuth which we address here is as follows:

Problem: Assume an insertion $I_{1}$ or $I_{2}$ occurs with probability $p$, and a deletion $D_{1}$ or $D_{2}$ occurs with probability $q$ :

$$
\operatorname{Pr}\left(I_{1}\right)=\operatorname{Pr}\left(I_{2}\right)=p ; \operatorname{Pr}\left(D_{1}\right)=\operatorname{Pr}\left(D_{2}\right)=q \text {. }
$$

with $p+q=\frac{1}{2}$. Let $(x, y)$ denote the absorbing state reached. What is the dependence of $\max (x, y)$ on $p$ and $m$ ? What is the asymptotic form of $\max (x, y)$ for fixed $p$ as $m$ tends to $\infty$ ?

The quantity $\max (x, y)$ is a measure of the efficiency of the storage allocation scheme, as we saw already. Knuth's model is nothing but a Markov chain (see Figure 1) with number of states equal to

$$
\frac{(m+1)(m+2)}{2}
$$

so that the probabilities of hitting a given absorbing state can always be determined numerically, for any given value of $m$, by inverting a matrix of dimension $O\left(\mathrm{~m}^{2}\right)$, a process that itself consumes a rather exorbitant $O\left(\mathrm{~m}^{6}\right)$ time. It is both impractical and uninformative. On the other hand, an asymptotic analysis gives results that are fairly simple to interpret and constitute, as we shall see later good numerical approximations even for relatively small values of $m$ (like $m=16$ or 32).

Let $A_{p}(m)$ denote the expected value of $\max (x, y)$ under Knuth's probabilistic model. Depending on the value of $\boldsymbol{p}$, rather different behaviours of $A_{p}(m)$ occur:

1. $p=\frac{1}{2}$ : no deletion occurs. The system reaches an absorbing state in $m$ steps exactly. Knuth [7, ex. 2.2.2.12], has shown that in this case:

$$
A_{p}(m)=\frac{m}{2}+\sqrt{\frac{m}{2 \pi}}+O\left(m^{-1 / 2}\right) \text {. }
$$

2. $\frac{1}{4}<p<\frac{1}{2}$ : we call this the expanding case since then $p>q$. The system is expected to reach an absorbing state in $O(m)$ steps. Yao [11] has proved for the expanding case that

$$
A_{p}(m)=\frac{m}{2}+\sqrt{\frac{m}{2 \pi(4 p-1)}}+O\left(\frac{\log ^{2} m}{\sqrt{m}}\right) .
$$

Yao's argument is in essence that after $\log m$ operations, the probability for one of the stacks to return to an empty state is asymptotically 0 , so that the analysis reduces to that of a much simpler random walk with only one absorbing barrier. Yao's argument also shows that the distribution of absorbing states is closely centered around $(m / 2 ; m / 2)$, the expected deviation being $O\left(m^{1 / 2}\right)$.

3. $p=\frac{1}{4}$ : the system is slowly expanding, and we call this the metastable case. In analogy to one-dimensional walks, see e.g. [9], we expect it to reach absorption in $O\left(m^{2}\right)$ steps. We shall prove that there: 


$$
A_{p}(m) \sim 0.67526 \ldots m
$$

so that the largest stack has size slightly over $\frac{2}{3} m$. Under this stack usage model, memory sharing proves quite efficient. We shall prove further that the probability for the system to reach state $(\lambda m ;(1-\lambda) m)$ approaches a limiting distribution (when $m$ gets large) with density $4\left(\frac{1}{2}+f(\lambda)+f(1-\lambda)\right)$, where $f(\lambda)$ admits the pseudo-Fourier expansion:

$$
f(\lambda)=\sum_{j=1}^{\infty} \cos j \lambda \pi \frac{\sinh j \lambda \pi}{\sinh j \pi} .
$$

The limiting distribution of stopping times is also characterised in terms a sort of bivariate theta function.

4. $0<p<\frac{1}{4}$ : the system is contracting since then $p<q$. It is expected to reach an absorbing state in $O\left(\left(\frac{q}{p}\right)^{m}\right)$ steps. We prove the somewhat surprising result that in this case the limiting distribution of absorbing states is uniform, so that:

$$
A_{p}(m) \sim \frac{3}{4} m
$$

and memory sharing is a real advantage. We also prove that the normalised stopping times obey a limiting exponential distribution of a simple form. Note that for a fixed value of $m$, as $\boldsymbol{p} \rightarrow 0$. Knuth states that the probability distribution of $x$ tends to a quasi-uniform distribution so that then again:

$$
\lim _{p \rightarrow 0} A_{p}(m) \sim \frac{3 m}{4} .
$$

Thus rather different situations occur depending on the relative position of $p$ and $q$. Note that Yao's proof of case (ii) is based on probabilistic approximations with the expanding character of the random walk "built in" the combinatorial reasoning, so that it cannot be extended to cover the general problem. In contrast in this paper, we propose to give analytic expressions for the probability distribution of absorbing states, from which precise asymptotic expansions may be obtained.

Plan of the paper: The analysis of the shared memory allocation scheme decomposes into simpler and simpler combinatorial subtasks which we shall explore in the subsequent sections:

(i) The problem of estimating the probability distribution of absorbing states reduces to the problem of counting trajectories (walks) in a triangular subset of the integer lattice $\mathbf{N} \times \mathbf{N}$; the probabilities are related in a simple way to the generating functions of path counts.

(ii) Using an adequate extension of the reflection principle of Andre (see e.g. [2]) the counting of walks in a triangle is reduced to a 2 -dimensional counting of walks in a square (with adequate boundary conditions).

(iii) Walks in a square are in turn decomposed as shuffles of 1dimensional walks over an interval (again with suitable boundary conditions). an operation which corresponds to products of exponential generating functions.

(iv) Walks over an integer interval have generating functions naturally expressible in terms of continued fractions $[1,3]$ and we treat them using the 
general theory set up in [3].

The expressions obtained from this chain of combinatorial reductions are then subjected to asymptotic analysis. Essentially, this involves studying the asymptotic distribution of poles of a class of rational functions related to Tchebycheff polynomials. From there, limiting distributions of absorbing states and absorption time are obtained.

Radically different behaviours occur in the 3 cases: expanding, metastable and contracting. To save space, we shall not develop the treatment of the expanding case corresponding to Yao's result.

\section{WALKS OVER AN INTEGER INTERVAL.}

Consider the graph $\Gamma_{m}$ whose set of vertices is the integer interval $[1 . m]$ and set of edges is:

$$
\{(x, x+1) \mid 0 \leq x<m\} \cup\{(x, x-1) \mid 0<x \leq m\} \cup\{(0,0) ;(m, m)\} .
$$

The 3 categories of edges in (1) are called respectively forward edges, backward edges and loops. We let $U_{k, n, t}$ denote the set of paths in $\Gamma_{m}$ from vertex 0 to vertex $k$ comprising a total of $n$ steps, out of which $t$ are loops. The problem is to estimate $U_{k, n, t}=\operatorname{card} U_{k, n, t}$.

The approach uses generating functions. Let

$$
U_{k}(x, u)=\sum_{n, t \geq 0} U_{k, n, t} x^{n} u^{t}
$$

One has:

Lemma 1: The generating function of the $U_{0, n, t}$ has the continued fraction expansion:

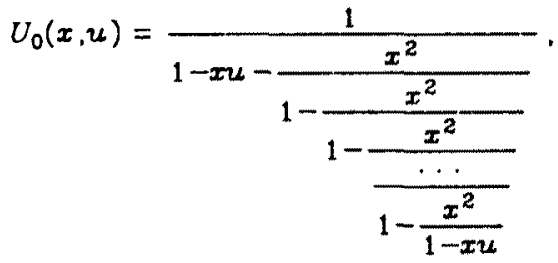

where the number of $x^{2}$ in the fraction is equal to $m$.

Let $V^{\langle m\rangle}$ denote the generating function of path in the graph $\Gamma_{m}$ that do not traverse the loop around $(0,0)$. We find, using techniques akin to those of [1] and [3]:

$$
V^{<m>}(x, u)=\frac{F_{m+1}(x)-x u F_{m}(x)}{F_{m+2}(x)-x u F_{m+1}(x)}
$$

where the $F_{m}$ are the Fibonacci polynomials:

$$
F_{0}(x)=0 ; F_{1}(x)=1 ; \quad F_{m+2}(x)=F_{m+1}(x)-x^{2} F_{m}(x) \text {. }
$$

From there, we can determine the quantities $U_{k}$, for general $k$; decomposing paths according to their last passages at levels $0,1,2, \cdots$, we find:

$$
U_{k}(x, u)=U_{0}(x, u) x V^{\langle m-1\rangle}(x, u) x V^{\langle m-2\rangle}(x, u) \cdots x V^{\langle m-k\rangle}(x, u)
$$


so that $U_{k}(x, u)$ is equal to:

$$
\frac{x^{k}}{1-x u-x^{2} V^{<m-1>}(x, u)} V^{<m-1>}(x, u) V^{<m-2>}(x, u) \cdots V^{<m-k>}(x, u) .
$$

After simplifications, we obtain:

Proposition 1a: The generating functions $U_{k}(x, u)$ satisfy:

$$
U_{k}(x, u)=x^{k} \frac{F_{m-k+1}(x)-x u F_{m-k}(x)}{F_{m+2}(x)-2 x u F_{m+1}(x)+x^{2} u^{2} F_{m}(x)} .
$$

There is an obvious connection between Fibonacci polynomials and Tchebycheff polynomials. Comparing their recurrence relations shows that:

$$
F_{m}\left(\frac{1}{2 \cos \vartheta}\right)=\left(\frac{1}{2 \cos \vartheta}\right)^{m-1} \frac{\sin m \vartheta}{\sin \vartheta}
$$

Proposition $1 b$ : The generating functions $U_{k}(x, u)$ satisfy:

$$
U_{k}\left(\frac{1}{2 \cos \vartheta}, u\right)=2 \cos \vartheta \frac{\sin (m-k+1) \vartheta-u \sin (m-k) \vartheta}{\sin (m+2) \vartheta-2 u \sin (m+1) v+u^{2} \sin m \vartheta}
$$

This last form is necessary in order to determine the inverse Laplace transform of $U_{k}$ that will be required later. To prepare for that task, we compute here the partial fraction expansion of $U_{k}(x, u)$. For convenience reasons, we first set:

Definition 1: Let $E(\varphi)$ be a function of $\varphi$, such that: $\frac{E(\varphi)}{\sin \varphi}=F(\cos \varphi)$, for some polynomial $F(z)$. A multiset $\Phi$ of roots of equation $E(\varphi)=0$ is said tr be a principal set of roots iff the multiset $Z=\{\cos \varphi\}$ is the multiset of roots of $P(z)$ each appearing with its multiplicity.

Proposition 1c: For a fixed $u>0$, and $m$ large enough, the poles of $U_{k}(x, u)$. taken as a function of $x$, are all simple. Function $U_{k}$ admits the partial fraction decomposition:

$$
U_{k}(x, u)=-\sum_{\varphi \in \Phi} \frac{c_{k}(\varphi)}{(1-2 x \cos \varphi)},
$$

where $\Phi$ is a set of principal roots of equation:

$$
E(\varphi) \equiv \sin (m+2) \varphi-2 u \sin (m+1) \varphi+u^{2} \sin m \varphi=0
$$

and $c_{k}(\varphi)$ is given by:

$$
c_{k}(\varphi)=2 \sin \varphi \frac{\sin (m-k+1) \varphi-u \sin (m-k) \varphi}{(m+2) \cos (m+2) \varphi-2(m+1) u \cos (m+1) \varphi+m u^{2} \cos m \varphi}
$$

for $\varphi \neq 0$, with $c_{k}(\varphi)=-\frac{1}{m+1}$ if $\varphi=0$.

The proof relies on the trigonometrical form of $U_{k}$. The quantity $E(\varphi) / \sin (\varphi)$ represents a polynomial of degree $m+1$ in $\cos \varphi$ that has, for general $u, m+1$ distinct roots. In particular, for the special case when $u=1$, for even $m$ the roots of $E(\varphi)$ are simply 


$$
\Phi=\left\{\frac{j \pi}{m+1} \mid \quad 0 \leq j \leq m\right\} .
$$

a fact to be used in later analyses of the metastable case.

\section{WALKS IN THE SQUARE}

We now consider walks on a square lattice. Define $\Lambda_{m}$ to be the (multi-) graph whose set of vertices is:

$$
\left\{\left[x_{1}, x_{2}\right] \mid 0 \leq x_{1}, x_{2} \leq m\right\}
$$

and set of edges:

$$
\begin{aligned}
& \left\{\left(\left[x_{1}, x_{2}\right],\left[\left|x_{1}+1\right|^{-}, x_{2}\right]\right)\right\} \cup\left\{\left(\left[x_{1}, x_{2}\right],\left[\left|x_{1}-1\right|^{+}, x_{2}\right]\right)\right\} \\
& \cup\left\{\left(\left[x_{1}, x_{2}\right],\left[x_{1},\left|x_{2}+1\right|^{-}\right]\right)\right\} \cup\left\{\left(\left[x_{1}, x_{2}\right],\left[x_{1},\left|x_{2}-1\right|^{+}\right]\right)\right\}
\end{aligned}
$$

where $x_{1}, x_{2}$ are in the integer interval [1..m], and

$$
|y|^{+}=\text {if } u>0 \text { then } u \text { else } 0
$$

We let $Q_{k_{1}, k_{2, n, t}}$ denote the number of paths connecting the origin $[0,0]$ to the point $\left[k_{1}, k_{2}\right]$ in $n$ steps, out of which $t$ are loops. We have:

Lemma 2: The exponential generating function of the $Q_{k_{1}, k_{2}, n, t}$ satisfies:

$$
\hat{Q}_{k_{1}, k_{2}}(x, u) \equiv \sum_{n, t \geq 0} Q_{k_{1}, k_{2}, n, t} u^{t} \frac{x^{n}}{n !}=\hat{U}_{k_{1}}(x, u) \hat{U}_{k_{2}}(x, u)
$$

where the $\hat{U}_{k}$ are the exponential generating functions of the numbers of 1 dimensional walks:

$$
\hat{U}_{k}(x, u)=\sum_{n, t \geq 0} U_{k, n, t} u^{t} \frac{x^{n}}{n !} .
$$

Proof: The proof corresponds to the fact that a 2 -dimensional walk in the square decomposes into the "shuffle" of two 1-dimensional walks on the line, whence the recurrence:

$$
Q_{k_{1}, k_{2}, n, t}=\sum_{\substack{n_{1}+n_{8}=n \\
t_{1}+t_{2}=t}}\left(\begin{array}{c}
n \\
n_{1}
\end{array}\right) U_{k_{1}, n_{1}, t_{1}} U_{k_{2}, n_{2}, t_{2}}
$$

Combining Lemma 2 with the result of Proposition $1 \mathrm{c}$ permits to express the ordinary generating function of the $Q_{k_{1}, k_{2}, n, t}$ :

Proposition 2: The ordinary generating function of the $Q_{k_{1}, k_{2}, n, t}$ is given by:

$$
Q_{k_{1}, k_{2}}(x, u) \equiv \sum_{n, t \geq 0} Q_{k_{1}, k_{2}, n, t} u^{t} x^{n}=\sum_{\varphi_{1}, \varphi_{z} \in \Phi} \frac{c_{k_{1}}\left(\varphi_{1}\right) c_{k_{2}}\left(\varphi_{2}\right)}{1-2 x\left(\cos \left(\varphi_{1}\right)+\cos \left(\varphi_{2}\right)\right)}
$$

Proof: From Proposition 1c, we see that the exponential generating function $\hat{U}_{k}(x, u)$ is:

$$
\hat{O}_{k}(x, u)=\sum_{\varphi \in \Phi} c_{k}(\varphi) e^{2 x \cos \varphi}
$$


since, by inverse Laplace transform, the exponential generating function corresponding to an element $(1-\alpha x)^{-1}$ is $\exp (\alpha x)$. Thus, by Lemma $2, \widehat{Q}$ has the expression:

$$
\widehat{Q}_{k_{1}, k_{2}}(x, u)=\sum_{\varphi_{1}, \varphi_{2} \in \Phi} c_{k_{1}}\left(\varphi_{1}\right) c_{k_{2}}\left(\varphi_{2}\right) e^{2 x\left(\cos \varphi_{1}+\cos \varphi_{2}\right)} .
$$

and $Q$ is obtained by transforming $\widehat{Q}$ by means of a Laplace transform: each element $\exp (\beta x)$ in the above expression transforms back to $(1-\beta x)^{-1}$.

\section{WALKS IN A TRIANGLEE AND THE PROBABILITY DISTRIBUTION OF ABSORBING STATES}

Andre's reflection principle has been originally developed in connection with 1-dimensional walks or ballot sequences.

Definition: A class of walks over the integer lattice defined by a set of elementary steps $\Sigma$ is said to satisfy the reflection principle for a straight line $\Delta$ if the following isomorphism holds, for all points $A, B$ :

$$
\operatorname{Path}_{n}^{\geq \Delta}[A, B] \sim \operatorname{Path}_{n}[A, B], \operatorname{Path}_{n}\left[\operatorname{sym}_{\Delta}(A), B\right] \text {, }
$$

where $\operatorname{Path}_{n}[X, Y]$ is the set of paths relating $X$ to $Y$ in $n$ steps, Path ${ }_{n}^{2 \Delta}$ denotes the subset of those paths that do not touch $\Delta$, and $\operatorname{sym} \Delta(A)$ is the symmetrical point of $A$ with respect to $\Delta$.

The interest of this principle is to reduce the problem of enumerating paths (with set of steps $\Sigma$ ) that do not touch a given line to enumerating to sets of unconstrained paths, usually a simpler combinatorial problem. To ensure its applicability, we essentially need that $\Sigma$ be closed by symmetry with respect to $\Delta$. Indeed, in this case, each path from $A$ that touches $\Delta$ has a first contact step (starting from $A$ ), and can be bijectively mapped onto a path relating $\operatorname{sym}_{\Delta}(A)$ by reflecting (by symmetry w.r.t. $\Delta$ ) all the steps until that first contact.

Thus the set of walks in the integer lattice with set of steps North, South, East, West satisfies the reflection principle for any line parallel to the second diagonal passing through one of the lattice points. A short reflection shows that the principle also applies to the set of walks in the square when $\Delta$ is the line of equation $x+y=m$.

We now define the quantities $T_{k_{1}, k_{2}, n, t}$ when $k_{1}+k_{2}<m$ to be the number of walks in the square (i.e. the graph $\Lambda_{m}$ ) of length $n$ comprising $t$ loops connecting the origin $(0,0)$ to the point $\left(k_{1}, k_{2}\right)$ that do not touch the line $x+y=m$. It proves convenient to extend the definition of the $T_{k_{1}, k_{2}}$ to the situation where $k_{1}+k_{2}=m$. In that case, we let $T_{k_{1}, k_{2}, n, t}$ denote the number of walks with terminal point $\left(k_{1}, k_{2}\right)$ which apart from their terminal point do not have any point on the diagonal $x+y=m$.

Such walks are called here triangular walks. From the previous argument, we obtain:

Proposition 3: The ordinary generating function of triangular walks defined by 


$$
T_{k_{1}, k_{2}}(x, u)=\sum_{n, t \geq 0} T_{k_{1}, k_{2}, n, t} u^{t} x^{n}
$$

satisfies when $k_{1}+k_{2}<m$ :

$$
T_{k_{1}, k_{2}}(x, u)=Q_{k_{1}, k_{2}}(x, u)-Q_{m-k_{2}, m-k_{1}}(x, u) .
$$

and for $k_{1}+k_{2}=m$ :

$$
T_{k_{1}, k_{2}}(x, u)=x\left(Q_{k_{1}-1, k_{2}}(x, u)+Q_{k_{1}, k_{2}-1}(x, u)-Q_{k_{1}+1, k_{2}}(x, u)-Q_{k_{1}, k_{2}+1}(x, u)\right) .
$$

We can now derive the probability distribution of absorbing states under Knuth's probabilistic model:

Lemma 3: The probability $\pi_{k_{1}, k_{2}}$ of reaching the absorbing state $\left(k_{1}, k_{2}\right)$ (with $k_{1}+k_{2}=m$ ) is equal to:

$$
\left.\pi_{k_{1}, k_{2}}=\left(\frac{p}{q}\right)^{m / 2} T_{k_{1}, k_{2}}(p q)^{1 / 2},\left(\frac{q}{p}\right)^{1 / 2}\right) .
$$

Proof: Observe that any path of length $n$ with $t$ loops is formed with $N, S, E, W$ steps going North, South, East, West respectively, and it has probability:

$$
p^{N+E} q^{H+S} q^{t}=(p q)^{n / 2}\left(\frac{q}{p}\right)^{t / 2}
$$

Whence our first theorem:

Theorem 1: [General Absorption Probabilities Theorem] The probability of reaching absorption in state $\left(k_{1}, k_{2}\right)$ satisfies:

$$
\pi_{k_{1}, k_{2}}=(p q)^{1 / 2}\left(\frac{p}{q}\right)^{m / 2} \sum_{\varphi_{1}, p_{2} \in \Phi}^{+} \frac{\left(c_{k_{1}-1}-c_{k_{1}+1}\right) c_{k_{2}}+c_{k_{1}}\left(c_{k_{2}-1}-c_{k_{2}+1}\right)}{1-2 x\left(\cos \varphi_{1}+\cos \varphi_{2}\right)} .
$$

where $x=(p q)^{1 / 2}, u=\left(\frac{q}{p}\right)^{1 / 2} . \Sigma^{+}$is to be interpreted as a sum with $\varphi_{1}, \varphi_{2}$ not both $0 . \Phi$ is a set of principal roots of equation:

$$
\sin (m+2) \varphi-2 u \sin (m+1) \varphi+u^{2} \sin m \varphi=0
$$

and $c_{k} \equiv c_{k}(\varphi)$ is $\left(c_{k_{1}} \equiv c_{k_{1}}\left(\varphi_{1}\right), c_{k_{2}} \equiv c_{k_{2}}\left(\varphi_{2}\right)\right)$ :

$$
c_{k}(\varphi)=2 \sin \varphi \frac{\sin (m-k+1) \varphi-u \sin (m-k) \varphi}{(m+2) \cos (m+2) \varphi-2(m+1) u \cos (m+1) \varphi+m u^{2} \cos m \varphi}
$$

with $c_{k}(\varphi)=-\frac{1}{m+1}$ if $\varphi=0$.

\section{THE METASTABLE CASE}

What we call here the metastable case is the situation where $p=q=1 / 4$. In this case, the expressions of Theorem 1 simplify appreciably since the set $\Phi$ is for even $m$ :

$$
\Phi=\left\{\frac{j \pi}{m+1} \mid 0 \leq j \leq m\right\}
$$


Whence, an adapted form of Theorem 1 in the metastable case:

Corollary 1: In the metastable case, the absorption probabilities are given by:

$$
\pi_{k_{1}, k_{2}}=A_{k_{1}}^{(m)}+A_{k_{2}}^{(m)}+B_{k_{1}, k_{2}}^{(m)} \text {. }
$$

where

$$
\begin{gathered}
A_{k}^{(m)}=\frac{2}{(m+1)^{2}} \sum_{j=1}^{m} \frac{\sin ^{2} \frac{j \pi}{m+1} \sin \frac{j \pi}{2 m+2}}{\left(1-\cos \frac{j \pi}{m+1}\right)^{2}} \sin \left(\left(k+\frac{1}{2}\right) \frac{j \pi}{m+1}\right), \\
B_{k_{1}, k_{2}}^{(m)}=\frac{4}{(m+1)^{2}} \sum_{j_{1}=1 j_{2}=2}^{m} \sum_{\left(1-\cos \frac{j_{1} \pi}{m+1}\right)\left(1-\cos \frac{j_{2} \pi}{m+1}\right)\left(1-\frac{1}{2}\left(\cos \frac{j_{1} \pi}{m+1}+\cos \frac{j_{2} \pi}{m+1}\right)\right)}^{\sin \frac{j_{1} \pi}{m+1} \sin \frac{j_{1} \pi}{2 m+2} \sin \frac{j_{2} \pi}{m+1} \sin \frac{j_{2} \pi}{2 m+2}} \\
{\left[\sin \frac{j_{1} \pi}{m+1} \sin \left(k_{1}+\frac{1}{2}\right) \frac{j_{1} \pi}{m+1} \cos \left(k_{2}+\frac{1}{2}\right) \frac{j_{2} \pi}{m+1}+\sin \frac{j_{2} \pi}{m+1} \sin \left(k_{2}+\frac{1}{2}\right) \frac{j_{2} \pi}{m+1} \cos \left(k_{1}+\frac{1}{2}\right) \frac{j_{1} \pi}{m+1}\right]}
\end{gathered}
$$

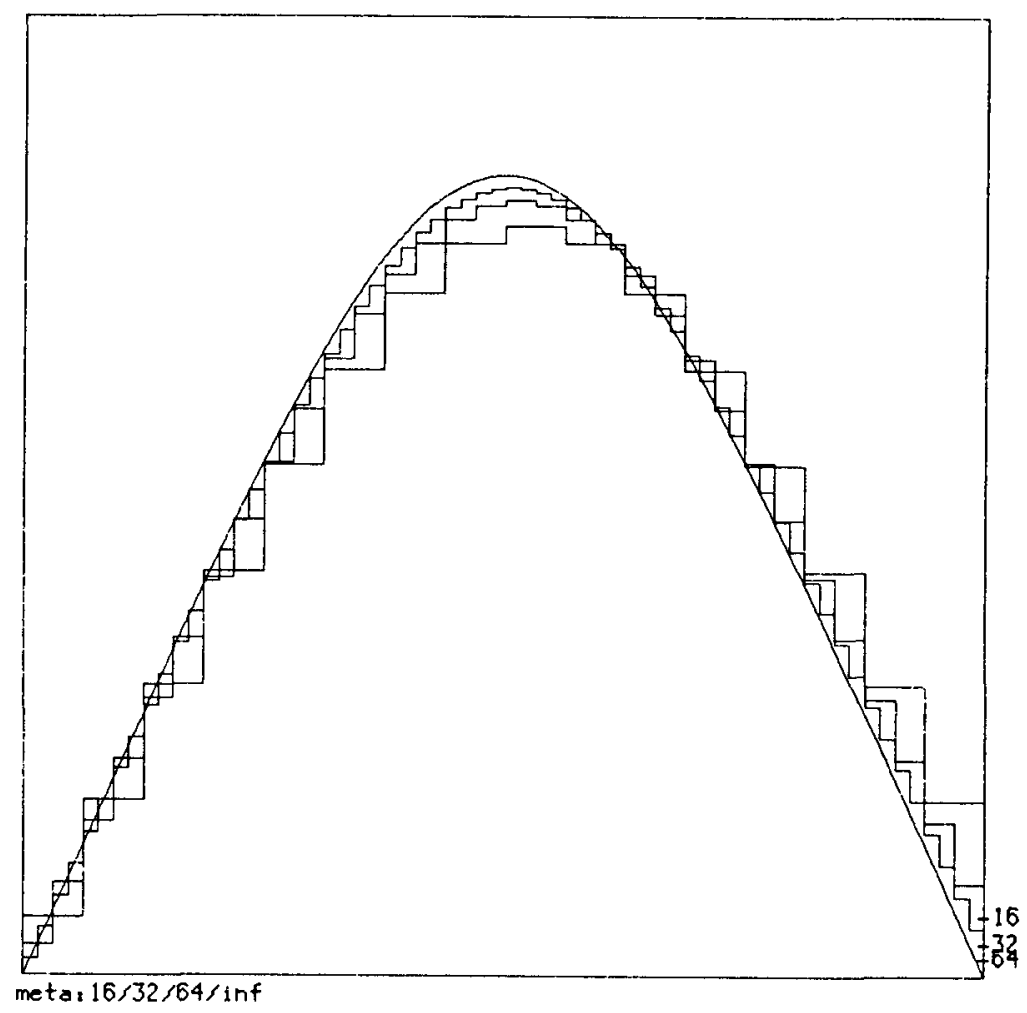

Figure 2: Convergence to the limit distribution (Theorem 2) of the exact distributions computed by means of Corollary 1 , for $m=16.32,64$.

This expression, though rather complicated, lends itself to easy numerical evaluation. Furthermore, it can be asymptotically analysed for large $m$. The dominant contribution comes from small values of $j_{1} j_{1}, j_{2}$ in the 
expressions of $A, B$. We can justify, for large $m$, the natural substitutions:

$$
\sin \frac{j \pi}{m+1} \rightarrow \frac{j \pi}{m} ; 1-\cos \frac{j \pi}{m+1} \rightarrow \frac{\dot{2}^{2} \pi^{2}}{2 m^{2}} \text {. }
$$

then extend the sums in Corollary 2 to indices $j, j_{1}, j_{2}$ going to $+\infty$.

In the process, there appear Fourier expansions of the periodic continuation of function $\frac{\pi}{2}-x$ as well as sums that can be reduced $[10, p .190]$ using the Fourier expansion:

$$
\sum_{j \geq 1} \frac{j \sin j x}{a^{2}+j^{2}}=\frac{\pi}{2} \frac{\sinh }{\sinh x} \frac{a(\pi-x)}{.}
$$

From these computations, we get:

Theorem 2: [Asymptotics of the metastable case] Assume $\lambda$ ranges over any fixed subinterval $[\alpha ; \beta]$ of $[0 ; 1]$. Then in the metastable case $(p=q=1 / 4)$, as $m$ tends to infinity in such $\alpha$ way that $\lambda . m$ is an integer, the absorption probabilities tend uniformly to a limiting distribution with a continuous density:

$$
\lim _{m \rightarrow \infty} m \cdot \pi \lambda m,(1-\lambda) m=4\left[\frac{1}{2}+f(\lambda)+f(1-\lambda)\right]
$$

where $f(x)$ is the function:

$$
f(x)=\sum_{n \geq 1} \cos n \pi x \frac{\sinh n \pi x}{\sinh n \pi} .
$$

The expected size of the largest stack when the shared memory algorithm stops satisfies:

$$
\lim _{m \rightarrow \infty} \frac{1}{m} A_{1 / 4}(m) \equiv \alpha\left(\frac{1}{4}\right)=\frac{7}{8}-2 \int_{0}^{1 / 2}(f(x)+f(1-x)) d x .
$$

and the constant $\alpha(1 / 4)$ evaluates numerically to: 0.67526 .

\section{THE CONTRACTING CASE}

In the contracting case where $p<q$, the set $\Phi$ of principal roots of $E(\varphi)$ no longer has the simple form of regularly spaced points. Rewriting the equation $E(\varphi)=0$ under the form:

one can prove:

$$
\frac{\sin (m+1) \varphi}{\sin m \varphi}=\frac{u^{2}-1}{2(u-\cos \varphi)}
$$

Lemma 4: When $p<q$, for $m$ large enough, a set $\Phi$ of principal roots of $E(\varphi)$ is formed of:

a. $(m-1)$ roots that separate the regularly spaced points:

$$
\frac{\pi}{m} ; \frac{2 \pi}{m} ; \frac{3 \pi}{m} ; \cdots \frac{(m-1) \pi}{m} ; \frac{m \pi}{m}
$$


b. Two complex roots $\varphi_{1}$ and $\varphi_{2}$, called dominant roots, satisfying:

$$
\cos \varphi_{1}, \cos \varphi_{2}=\frac{1}{2}\left(u+\frac{1}{u}\right)+O\left(u^{-m}\right)
$$

where $u=\left(\frac{q}{p}\right)^{1 / 2}>1$.

It is actually these two complex roots that dictate the asymptotic behaviour of the absorption probabilities. From detailed asymptotic expansions (obtained with the help of the MACSYMA system) of the dominant roots and corresponding expressions of Theorem 1, we get:

Theorem 3: [Asymptotics of the Contracting Case] Assume $\lambda$ ranges over any fixed subinterval $[\alpha ; \beta]$ of $[0 ; 1]$. Then in the contracting case $(p<q)$, as $m$ tends to infinity in such a way that $\lambda . m$ is an integer, the absorption probabilities converge uniformly to the uniform distribution:

$$
\lim _{m \rightarrow \infty} m \cdot \pi_{\lambda m,(1-\lambda) m}=1
$$

The expected size of the largest stack when the shared memory algorithm stops satisfies:

$$
\lim _{m \rightarrow \infty} \frac{1}{m} A_{p}(m) \equiv \alpha(p)=\frac{3}{4}
$$

Thus the largest stack has size approximately $0.75 \mathrm{~m}$ when absorption is reached. The really surprising fact is that the limitir. distribution of absorption states is uniform in that case, for fixed $\boldsymbol{p}$. (Note: this is quite different from the "not difficult" result cited by Knuth that concerns the case where $p \rightarrow 1$ ).

\section{STOPPING TIMES}

Let $W_{p}(m)$ be the random variable denoting the time when the shared memory algorithm stops, having run short of memory. As indicated in the introduction, knowledge of this parameter is of interest if shared memory allocation is to be compared to separate memory allocation. From previous methods, we can prove:

Theorem 4: [Waiting Times: Metastable Case] In the metastable case $(p=q=1 / 4)$, the stopping time has for large $m$ an expectation which is $O\left(m^{2}\right)$ and it obeys a limiting bivariate theta distribution.

For lack of space, we refrain from giving here the complete expressions of the limiting distribution and the implied constant in the $O\left(m^{2}\right)$ result.

Theorem 5: [Waiting Times: Contracting Case] In the contracting case $(p<q)$, the normalised stopping time:

$$
W_{p}^{*}(m)=\frac{1}{K} W_{p}(m) \cdot\left(\frac{\boldsymbol{p}}{\boldsymbol{q}}\right)^{m} .
$$

$K$ a constant for fixed $p$, has in the limit an exponential distribution: 


$$
\lim _{m \rightarrow \infty} \operatorname{Pr}\left[\alpha<W_{p}^{*}(m)<\beta\right]=e^{-\beta}-e^{-\alpha}
$$

In particular, in the contracting case, one expects to be able to process a number of request of the order of $O\left(\left(\frac{q}{p}\right)^{m}\right)$.

\section{NUMERICAI، ESTIMATES AND FINAL CONCLUSIONS}

In order to attain numerically accurate results, we have used the MAPLE symbolic computation system to determine exact rational expressions of stopping probabilities. Figure 3 displays the probability distributions of absorbing states for $m=20$ when $p=0.05,0.10, \cdots, 0.45$. (Note the graphs have been splined for easier readability.)

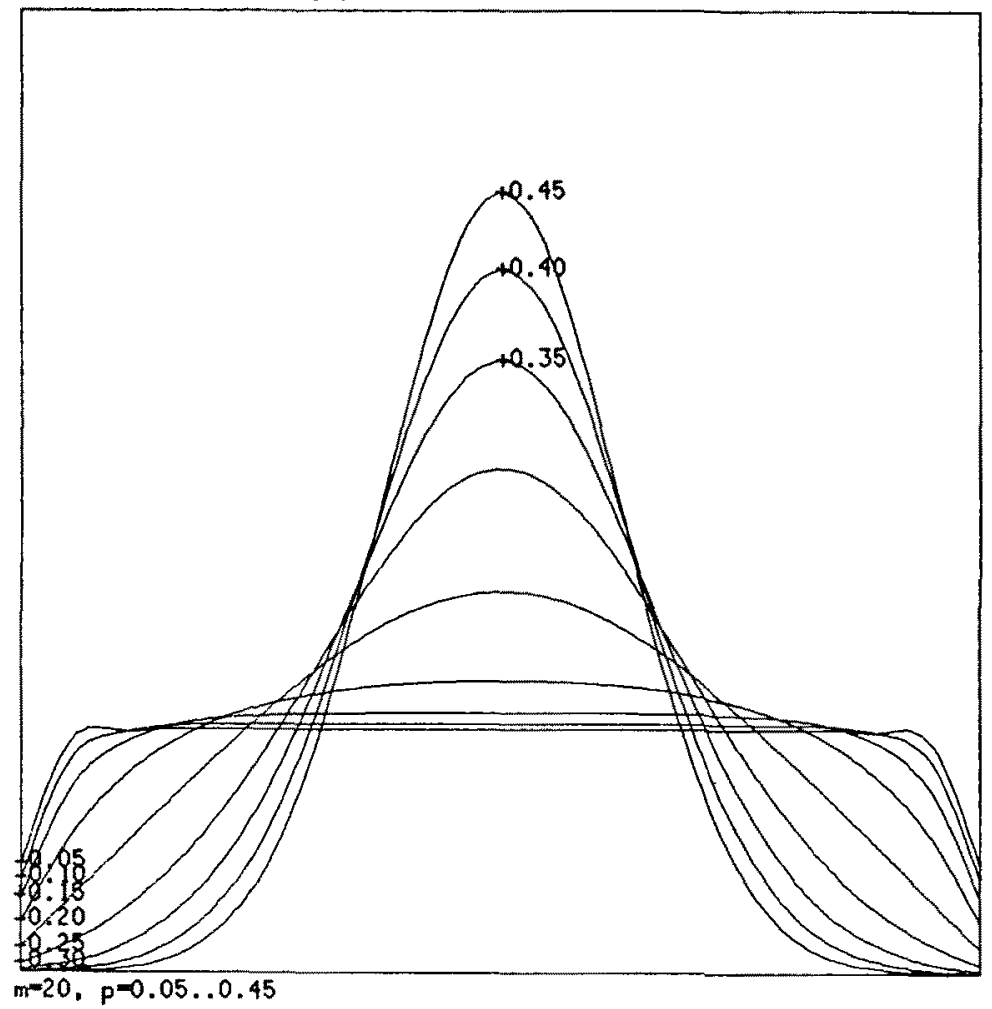

Figure 3: Absorption state probabilities for $m=20$ and $p=0.05(0.05) 0.45$ (after splining).

This provides a check for the exact expressions obtained for the metastable case whose convergence to a limit distribution was depicted in Figure 2. We also notice the flatness of the curves for low values of $p(0.5-0.15)$ which clearly confirms the uniformity of the limiting distribution in the contracting case. As an other example, when $m=32$, the probabilities of stopping 
at states $4,8,12,16$ when $p=1 / 6$ are respectively:

$0.0317708,0.0332356,0.0333272,0.0333326$.

(The computation took 15 hours of CPU time on a VAX $11 / 780$ !).

Thus these numerical computations confirm the usefulness and the validity of these asymptotic approximations even for small values of the memory size $m$. A summary of the results for this problem is displayed in Table 1.

\begin{tabular}{|c|c|c|c|c|}
\hline & \multicolumn{2}{|c|}{ Largest Stack } & \multicolumn{2}{|c|}{ Stopping Time } \\
\hline & Expect. & Distrib. & Expect. & Distrib. \\
\hline Expanding & $0.5 m$ & Dirac (Gaussian) & $O(m)$ & - \\
\hline Metastable & $0.6752 \mathrm{~m}$ & pseudo-Fourier & $O\left(m^{2}\right)$ & biv. theta \\
\hline Contracting & $0.75 \mathrm{~m}$ & uniform & $O\left(c^{m}\right)$ & exponential \\
\hline
\end{tabular}

Table 1: A summary of available results for the shared allocation algorithm.

The separate allocation algorithm can be analysed in an even simpler way (since it corresponds to walks in a square, $c f$. Sect. 3). It appears from Yao's results that not much difference results in the expanding case when $p>q$. Notice however that this situation does not seem to model real-life situations too well since then the allocation algorithms steadily progress to a situation where memory gets exhausted.

The metastable and contracting models seem more realistic. If $p=q=1 / 4$, we have seen that the algorithm based on sharing can accommodate fairly dissymmetrical files. That conclusion is even more true in the contracting case where one can further prove that the sharing algorithm will accept a number of requests exponentially larger than the separate allocation algorithm.

Therefore, an intuitively clear conclusion of our study could be:

"Share with somebody if you can!"

Acknowledgements: The author would like to thank A. Odlyzko, F. Baccelli, G. Fayolle, B. Chazelle, R. Sedgewick and J. Savage for several stimulating discussions during the elaboration of this work.

\section{References}

1. N. De Bruijn, D. E. Knuth, and S. O. Rice, "The Average Height of Binary Trees and Other Simple Trees," pp. 15-22 in Graph Theory and Computing, Academic Press, New-York (1972).

2. L. Comtet, Advanced Combinatorics, Reidel, Dordrecht (1974).

3. P. Flajolet, "Combinatorial Aspects of Continued Fractions," Discrete Math. 32 pp. 125-161 (1980).

4. P. Flajolet, J. Francon, and J. Vuillemin. "Sequence of Operations Ànalysis for Dynamic Data structures," J. of Alg. 1 pp. 111-141 (1980). 
5. J. Francon, "Histoires de Fichiers," RAIRO Inf. Theor, 12 pp. 49-62 (1979).

6. A. Jonassen and D. E. Knuth, "A Trivial Algorithm Whose Analysis Isn't," Stanford University Report STAN-CS-77-598 (1977).

7. D. E. Knuth, The Art of Computer Programming: Fundamental Algorithms, Addison Wesley, Reading, Mass (1968).

8. J. K. Percus, Combinatorial Methods, Springer Verlag (Applied Mathematical Sciences 4), New-York (1971).

9. F. Spitzer, Principles of Random Walk, Springer Verlag. New-York (1976). (2nd ed.).

10. E. T. Whittaker and G. N. Watson, A Course in Modern Analysis, Cambridge University Press (1927).

11. A. C. Yao, "An Analysis of a Memory Allocation Scheme for Implementing Stacks," SIAM J. Comput. 10(2) pp. 398-403 (1981). 